\section{$\$$ Research Square}

\title{
A Randomized Sham-controlled Trial of Manual Acupuncture for Infertile Women with Polycystic Ovary Syndrome
}

\section{Wen Pan}

Shanghai University of Traditional Chinese Medicine

\section{Feng-xing Li}

Shanghai University of Traditional Chinese Medicine

Qiao Wang

Shanghai University of Traditional Chinese Medicine

\section{Xiu-qi Yin}

Shanghai University of Traditional Chinese Medicine School of Nursing

\section{Zou-qin Huang}

Shanghai Pudong New Area Hospital of Traditional Chinese Medicine

\section{Yu-ming Yan}

Shanghai University of Traditional Chinese Medicine

\section{Ling Zhao ( $\nabla$ zl2@shutcm.edu.cn )}

Shanghai University of Traditional Chinese Medicine https://orcid.org/0000-0002-4017-8167

\section{Xue-yong Shen}

Shanghai University of Traditional Chinese Medicine

\section{Research}

Keywords: Polycystic Ovary Syndrome, Manual Acupuncture, Chinese Herbal Medicine, Infertility

Posted Date: August 9th, 2021

DOI: https://doi.org/10.21203/rs.3.rs-754196/v1

License: (c) (1) This work is licensed under a Creative Commons Attribution 4.0 International License. Read Full License

Version of Record: A version of this preprint was published at Integrative Medicine Research on December 1st, 2021. See the published version at https://doi.org/10.1016/j.imr.2021.100830. 


\title{
A Randomized Sham-controlled Trial of Manual Acupuncture for Infertile Women
}

\section{with Polycystic Ovary Syndrome}

Wen Pan ${ }^{1 \#}$, Feng-xing Li $^{1 \#}$, Qiao Wang ${ }^{1}$, Xiu-qi Yin ${ }^{2}$, Zou-qin Huang ${ }^{3}$, Yu-ming Yan $^{1}$, Ling Zhao $^{1 *}$, Xue-yong Shen ${ }^{1,2} *$

1. Acupuncture-moxibustion \&Tuina School, Shanghai University of Traditional Chinese Medicine, Shanghai 201203, China

2. Shuguang Hospital, Shanghai University of Traditional Chinese Medicine, Shanghai 201203, China

3. Shanghai Pudong New District Hospital of Traditional Chinese Medicine, Shanghai 201203, China

\# These authors contributed equally to this study and should be considered as co-first authors

* Corresponding authors: Ling Zhao: zl2@ shutcm.edu.cn, Xue-yong Shen:

$\underline{\text { sxy1@ } @ \text { shutcm.edu.cn }}$

\begin{abstract}
Background: To evaluate the effectiveness of acupuncture combined with herbal medicine among the infertile female with polycystic ovary syndrome (PCOS).

Method: A randomized, sham-controlled trial was conducted. A total of 86 women who were diagnosed as PCOS infertility for more than 1 year were randomly assigned to receive manual acupuncture (MA) or sham acupuncture (SA) twice per week for three menstrual cycles. Both groups received herbal medicine treatment. The evaluations were conducted at baseline, each menstruation, after three menstrual cycles and 24 weeks follow-up, including pregnancy rate, ovulation rate, sex hormones level, PCOS symptoms and Traditional Chinese Medicine (TCM) syndrome scores.
\end{abstract}


Results: Among 86 randomized patients, 79 (91.86\%) completed the trial. The pregnancy rate in the MA group was significantly higher than the SA group (46.34\% vs $18.42 \%$; $P=0.008)$. The ovulation rate of the MA group was higher than the SA group $(58.14 \%$ vs 45.74\%; $P=0.046)$. The improvement rate of PCOS score and testosterone level showed a statistical difference between the two groups $(P<0.05)$, others were no significant difference between the two groups. PCOS score and TCM symptom score of the two groups were both decreased after treatment $(P<0.001)$. Sex hormones level including E2, T, P, LH and LH/FSH were significantly lower after intervention in the MA group ( $P$ values were <0.05) compared with baseline, while only the progesterone level was reduced in the SA group $(P=0.008)$.

Conclusion: Manual acupuncture combined with herbal medicine may be clinically useful for infertile women with PCOS in improving pregnancy and ovulation rate.

Trial Registration: Chinese Clinical Trial Registry, ChiCTR1800014997.

\section{Keywords}

Polycystic Ovary Syndrome; Manual Acupuncture; Chinese Herbal Medicine; Infertility

\section{Background}

Polycystic ovary syndrome (PCOS) is a complex polygenic disease and is one of the most common endocrinological and reproductive disorders, $[1,2]$ threating $10 \%$ of women of child-bearing age.[3] It is characterized by biochemical hyperandrogenism, chronic 
anovulation and the presence of polycystic ovaries, [4] which cause more than $75 \%$ of an ovulatory infertility.[5] PCOS syndromes including obesity, impaired glucose tolerance, excessive body hair, acne, menstrual cycle disturbances, infertility and imbalance of hormones.[6] PCOS is complicated by depression, anxiety, et al, and posing a health hazard to women of childbearing age.[7, 8]

Clomiphene citrate (CC) has been used as the first-line treatment for an ovulatory infertility in women with PCOS, metformin and letrozole are also widely prescribed.[9, 10] Cumulative ovulation rates of $\mathrm{CC}$ are as high as $90 \%$, with pregnancy rate is 50 70\%.[11] But up to $40 \%$ of women who received treatment with clomiphene show little clinical improvement,[12] a relatively high multiple-pregnancy rate (3 8\%), and side effects such as mood changes, hot flushes,[13] dizziness, nausea, gastrointestinal symptoms, and symptoms associated with ovarian enlargement and ovulation,[14] even birth defects.[15] Acupuncture and TCM methods have a long history of using for gynecological diseases and fertility issues such as those associated with PCOS.[16, 17] However, available evidence is limited. The present study was aimed to estimate the efficacy of acupuncture combined with herbal medicine on ovulation rate, pregnancy rate and related hormones level in infertile women with PCOS.

\section{Materials and methods}

\subsection{Study design}

This investigation was a randomized, sham-controlled study. It was conducted 
between March 2018 and December 2019, in the outpatient clinics in Shuguang Hospital in Shanghai, China. The protocol was published and is available in the Supplement.[18] This study was registered on 27 February 2018 in the Chinese Clinical Trial Registry (ChiCTR1800014997). https://www.chictr.org.cn/showproj.aspx?proj=25583.

\subsection{Patients}

Eligible women were aged 20 to 40 years, and met the diagnosis criteria for both infertility and PCOS defined by the Rotterdam criteria proposed in 2003.[4] Besides, the husband was required to have normal semen examination results.

Women with any of the following conditions were excluded: hyperprolactinemia, adrenal or ovarian tumors, thyroid disease, Cushing syndrome, and other heart and kidney diseases; oral contraceptives and hormone drugs during the first month and other type of infertility; history of receiving acupuncture/moxibustion treatment within 2 months; use of other drugs that could affect the reproductive function or metabolism; Participation in another clinical study in the past two months.

\subsection{Randomization and blinding}

A total of 86 eligible participants were recruited and were randomly assigned at a 1:1 ratio to receive MA or SA treatment. Randomization sequence was generated using Excel by Dr. Cheng. Allocation concealment was ensured in an envelope. The envelopes were sealed, and the assignment records weren't disclosed until the end of the study. Ms. Wang enrolled participants, and Ms. Pan assigned participants to interventions. Patients were 
blinded after assignment to interventions. Acupuncturists could not be blinded to the treatment assignments given the nature of the interventions. Communication among participants was discouraged and avoided as they were treated in separate room. Therefore, in this trial, participants, outcome assessors, data collectors, and statisticians were blinded to the treatment allocation.

\subsection{Interventions}

Both groups received real herbal medicine treatment twice a day as a basic treatment. During the menstrual period, patients were orally administered the Taohong Siwu decoction. Non-menstrual period treatment, were based on pattern differentiation: for spleen-kidney yang deficiency, applied Bushen Tiaojing decoction and liver-kidney deficiency were given Guishao Dihuang decoction.

Two acupuncturists with a clinical experience of more than three years were responsible for the manipulation of $\mathrm{MA}$ and $\mathrm{SA}$ after training. Sterile, disposable acupuncture needles $(0.25 \times 40 \mathrm{~mm})$, blunt-tip needles $(0.25 \times 25 \mathrm{~mm}$, Wuxi Jiajian Medical Instrument Co. Ltd, China) and simple adhesive pads (for fixation) were used. Participants received three menstrual cycles of acupuncture treatment (twice per week) and Chinese herbal medicine (twice a day). They received acupuncture on-obligatory points, including RN4, bilateral EX-CA1, ST29, ST36 and SP6. The other points were chosen according to syndrome differentiation and the menstrual cycle. The potential acupoints include RN6, RN12, DU20, and bilateral ST25, KI3, KI6, LR3, SP10, PC6. 
Each patient was selected no more than 13 points. The use of additional acupoints other than the prescribed ones was not allowed. The semistandard prescriptions is based on the textbook "Acupuncture and Moxibustion" and previous clinical experience.[19] If the participant has evidence of pregnancy, she will no longer receive treatment.

During acupuncture treatment, adhesive pads were pasted on acupoints after sterilization. The acupoints were needled through the pad and 10-30mm into the skin (depend on location). were manipulated with even twirling, lifting, and thrusting to achieve the "DeQi" sensation (a sensation of soreness, numbness, distention, or radiating that indicates effective needling) or needling sensation once every 10 minutes for 30 minutes.[20] The treatment procedure in the SA group was identical in the MA group except not achieve DeQi sensation. [18]

\subsection{Outcome measures}

Participants were assessed at baseline, each menstruation, after three menstrual cycles of treatment, and 24 weeks follow-up after the completion of the treatment. The primary outcome was the pregnancy rate during the trial. Patient reported pregnancy or HCG positive in blood or urine, which was confirmed by B-ultrasound. The secondary outcomes included the ovulation rate observed through Basal body temperature (BBT) or B ultrasound every menstrual cycle; change from baseline in PCOS and TCM syndrome score (scoring was additive which higher values indicating increased severity); and change from baseline of the serum sex hormones level included estradiol $\left(\mathrm{E}_{2}\right)$, 
testosterone $(\mathrm{T})$, progesterone $(\mathrm{P})$, luteinizing hormone $(\mathrm{LH})$, follicle stimulating hormone (FSH). In addition, insulin resistance index (HOMA-IR $=$ fasting blood glucose $\times$ fasting insulin/22.5) was assessed at baseline and after treatment of three menstrual cycles. 24 weeks after the completion of the treatment, follow up performed through the telephone to ask if the participants were pregnant. Acupuncture-associated adverse events were recorded and reported to the study coordinators at each treatment session.

\subsection{Ethical considerations}

The ethics committee has been approved by Institutional Review Board of Shuguang hospital affiliated with Shanghai University of TCM (22 Dec 2017, Approval Number: 2017-569-52-01). All participants have signed written informed consent after the trial procedures were fully explained and they also consented to the publication of clinical results. The protocol was approved by the institutional ethics review board of Shuguang Hospital and was performed in accordance with the Declaration of Helsinki.

\subsection{Sample size calculation}

We designed the trial to determine whether there was a difference between the MA group and the SA group in terms of the pregnancy rate. According to the other studies,[21, 22] we hypothesized that the pregnancy rate would be $76 \%$ in the MA group and $40 \%$ in SA group. Assuming a two-side alpha of 0.05 , power of $90 \%$, and a $20 \%$ drop-out rate, a sample size of 36 would be needed for each group to detect a between-group difference. We expanded the sample size to 86 cases (43 cases per group) to increase the reliability of 
the study.

\subsection{Statistical analysis}

We performed a statistical analysis based on the intention-to-treat population, which includes participants who had a least 1 treatment and 1 primary outcome measure $(n=79)$. Missing data were completed by the last observed value. Continuous data were presented with mean and standard deviation $(\bar{x} \pm \mathrm{s})$ if they were normally distributed or with median (interquartile range, IQR) if they were abnormally distributed. Categorical variables were expressed as numbers and percentages. Chi-square tests were used to compare categorical variables, and 2 sample t-test or Wilcoxon rank sum test for continuous data, as appropriate. The variance analysis will be performed on the difference between the two groups and within the group. All reported $P$ values were two-sided and used a significance level of 0.05. All statistical analyses were performed using SPSS statistical software (version 21.0, International Business Machines Corporation, China).

\section{Results}

\subsection{Participants and baseline characteristics}

A total of 178 participants were invited to participate in the study, of whom 92 were excluded, and 86 were eligible and randomly assigned to the MA group or the SA group. 2 dropped out in MA group (1 case changed her job and 1 case had time inconvenience). 5 dropped out in SA group ( 2 cases had job changeds, 2 cases had time inconvenience and 1 
case unwilling to follow up). Seventy-nine participants (91.86\%) completed the study (Figure 1).

Baseline characteristics were similar between the groups (Table 1). No significant difference was found between the two groups in age, duration of illness, BMI, PCOS score, TCM score and serum sex hormones level. Patient characteristics were well balanced by randomized.

\subsection{Primary outcome}

During treatment, out of 79 patients were included in the statistical analysis, a total of 15 participants were successfully conceived, including 10 in the MA group and 5 in the SA group (Table 2). The pregnancy rate was $24.39 \%$ (10/41) in patients who received manual acupuncture combined with herbal medicine and $13.16 \%(5 / 38)$ in the sham acupuncture combined with herbal medicine group.

During the 6-month follow-up period, a further 11 patients reported pregnancy. There were 9 participants in the MA group with a pregnancy rate of $29.03 \%(9 / 31)$ and 2 in the sham acupuncture group with a pregnancy rate of $6.06 \%(2 / 33)(P=0.015)$. Thus, the pregnancy rate in the MA group was $46.34 \%$ (19/41) throughout the trial, significantly better than the $18.42 \%(7 / 38)$ in the SA group $(P=0.008)$.

\subsection{Secondary outcomes}

During the treatment period, the ovulation rate was $58.14 \%$ in the MA group, which 
was significantly better than $45.74 \%$ in the SA group $(P=0.046$, Table 3$)$. At the end of three menstrual cycles treatment, patients randomized to MA group had statistically significant differences in PCOS score and testosterone levels compared to the SA group, with greater improvements observed in the MA (Table 4).

After completion of treatment, compared the baseline, patients in MA group had statistically significant improvement in PCOS score, TCM score, estrogen $\left(\mathrm{E}_{2}\right)$, testosterone $(\mathrm{T})$, progesterone $(\mathrm{P})$, luteinizing hormone $(\mathrm{LH})$ level and the ratio of luteinizing hormone to follicle stimulating hormone, but no significant improvement in follicle stimulating hormone (FSH) level or insulin resistance index (HOMA-IR). Details are listed in Table 4. Patients of SA group showed statistically significant improvement in PCOS score, TCM score and progesterone level compared with baseline, while no significant changes were found in other hormone levels $(P>0.05)$. There were no significant adverse reactions in the two groups during the treatment period.

\section{Discussion}

This randomized sham-controlled trial found that manual acupuncture combined with herbal medicine was superior to sham acupuncture combined with herbal medicine for improving the pregnancy rate, ovulation rate and quality of life among infertile women with PCOS and that manual acupuncture provided some additional benefit over sham acupuncture. These findings support acupuncture combined with herbal medicine as an infertility treatment in patients with PCOS. 
Pregnancy is the ultimate evaluation indicator of infertility treatment. We found manual acupuncture combined with Chinese herbal medicine (MA group) for three menstrual cycles period showed significant efficacy in improving pregnancy rate and ovulation rate compared with SA group, and have a better long-term effect. Our findings are similar to previous reports.[23-25] In a systematic review reported a promising effect that Bushen Huохиe herbal medicine had advantages on increasing pregnancy rate, while the quality of evidence was relatively low.[26] Complementary and alternative medicine therapies, including TCM and acupuncture is increasingly widely used.[27] In this trial, we selected the acupuncture points according to literatures. [28-30]

According to the theory of TCM, syndrome differentiation and treatment is an important therapeutic principle. Treatment according to the phase of the menstrual cycle is also common in gynecological diseases. Patients received acupuncture treatment from the third day of menstruation to the third day of ovulation may took full advantages of acupuncture, promoted the development and discharge of follicles, and avoided the risk of acupuncture treatment during pregnancy.

Evidence from systematic reviews suggested that true acupuncture may improve ovulation and menstruation rates compared with no acupuncture.[31, 32] Effectiveness of acupuncture for PCOS is inconclusive due to insufficient overall evidence.[33] A randomized controlled trial demonstrated that acupuncture failed to treat the women with PCOS, [34] which was attracted wide attention and controversy.[35, 36] Acupuncture is a complex intervention associated with therapeutic gains.[37] Different treatment efficacy 
may be related to the treatment regimen, selection of acupoints, manipulation, treatment duration, frequency and qualification of the acupuncturist.[38] We considered these factors in the design of the trial. The compliance of the participants in the trial was high (91.86\%), maybe since these patients had a strong desire to get pregnant. No serious side effects were observed during the trial.

Peripheral blood examination showed that the manual acupuncture group could significantly reduce $\mathrm{E} 2, \mathrm{~T}, \mathrm{P}, \mathrm{LH}$ levels and $\mathrm{LH} / \mathrm{FSH}$ ratio in infertility patients with PCOS. However, only a statistically significant decrease in P level was observed in the SA group. Acupuncture may improve the hyperandrogenic state in PCOS patients, leading to improve ovulation and pregnancy rates. Studies have shown that acupuncture can reduce the LH/FSH ratio, decrease T, LH, and BMI levels, improve ovarian function, and can improve the local microcirculation and uterine wall environment in patients with polycystic ovary syndrome, which is conducive to implantation of fertile eggs, similar to Diane-35.[39, 40] Research has demonstrated that oral contraceptives can effectively reduce LH/FSH ratio and androgen levels, improve symptoms and restore ovulation to some extent, and reduce endometrial hyperplasia.[41]

Infertile PCOS patients show a tendency of the insulin resistance index improvement after acupuncture with no significant difference compare with the sham group. Both groups have similar effect in improving TCM symptoms may be due to the role of Chinese herbal decoction. While the improvement of clinical symptoms of manual acupuncture combined with herbal medicine is significantly better than that of the sham 
group. Thus provided evidence of acupuncture in the treatment of infertile PCOS patients.

The limitations of this study are its small sample size, and missing of an assessment of the blinding effectiveness and record of how many patients in the MA and SA groups reported achieving deqi. This trial was conducted in a single clinical trial center, and the imputation method for missing data that we used, the last observation carried forward method, may result in bias.

\section{Conclusion}

Manual acupuncture of three menstrual cycles combined with herbal medicine increased pregnancy rate and ovulation rate compared with sham acupuncture combined with herbal medicine among infertile women with PCOS. A larger sample of clinical trial is warranted to understand the long-term efficacy and the mechanism of action of acupuncture intervention.

\section{Abbreviations}

PCOS: Polycystic ovary syndrome; IR: Insulin resistance; MA: manual acupuncture; SA: Sham acupuncture; TCM: Traditional Chinese Medicine; CC: Clomiphene citrate; OHSS: Ovarian Hyper-stimulation Syndrome; RCTs: Randomized controlled trials; IRI: Insulin Resistance Index; HOMA-IR: insulin resistance index; $\mathrm{E}_{2}$ : estradiol; T: testosterone; P: progesterone; LH: luteinizing hormone; FSH: follicle stimulating hormone. 


\section{Declarations}

\section{Ethics approval and consent to participate}

The ethics committee has been approved by the Institutional Review Board of Shuguang hospital affiliated with Shanghai University of TCM (22 Dec 2017, Approval Number: 2017-569-52-01). All participants have signed written informed consent.

\section{Availability of data and materials}

All the data used to support the findings of this study are available from the corresponding authors upon reasonable request.

\section{Competing interests}

The authors declare that they have no competing interests.

\section{Funding}

This work was funded by the Pudong New Area Health Commission, Shanghai, China (Grant Code PDZY-2018-0610).

\section{Authors' contributions}

LZ and XYS: Conceived the idea and designed the trial. WP and QW: Participated in the planning of the project, analyses and interpretation of the results and wrote the manuscript. $\mathrm{ZQH}$ : Obtained funding and participated in the planning of the project. FXL and YMY: Participated both in statistical analyses. XQY: Provided the administrative, technical, or material support. All authors read and approved the final manuscript.

\section{Acknowledgements}

We would like to thank all the patients, nurses and professionals in Shuguang Hospital for their help and support.

\section{Consent to publish}

We declare that the Publisher has the Author's permission to publish the relevant contribution. 


\section{References:}

[1] Dumesic DA, Oberfield SE, Stener-Victorin E, Marshall JC, Laven JS, Legro RS. Scientific Statement on the Diagnostic Criteria, Epidemiology, Pathophysiology, and Molecular Genetics of Polycystic Ovary Syndrome. Endocrine reviews 2015;36(5):487-525.

[2] Norman RJ, Dewailly D, Legro RS, Hickey TE. Polycystic ovary syndrome. Lancet (London, England) 2007;370(9588):685-97.

[3] Yildiz BO, Bozdag G, Yapici Z, Esinler I, Yarali H. Prevalence, phenotype and cardiometabolic risk of polycystic ovary syndrome under different diagnostic criteria. Hum Reprod 2012;27(10):3067-73.

[4] Rotterdam EA-SPcwg. Revised 2003 consensus on diagnostic criteria and long-term health risks related to polycystic ovary syndrome (PCOS). Hum Reprod 2004;19(1):41-7.

[5] Gorry A, White DM, Franks S. Infertility in polycystic ovary syndrome: focus on low-dose gonadotropin treatment. Endocrine 2006;30(1):27-33.

[6] Pasquali R, Gambineri A, Pagotto U. The impact of obesity on reproduction in women with polycystic ovary syndrome. BJOG : an international journal of obstetrics and gynaecology 2006;113(10):1148-59.

[7] Azizi Kutenaee M, Amirjani S, Asemi Z, Taghavi SA, Allan H, Kamalnadian SN, et al. The impact of depression, self-esteem, and body image on sleep quality in patients with PCOS: a cross-sectional study. Sleep \& breathing $=$ Schlaf \& Atmung 2019.

[8] Bazarganipour F, Ziaei S, Montazeri A, Foroozanfard F, Kazemnejad A, Faghihzadeh S. Psychological investigation in patients with polycystic ovary syndrome. Health and quality of life outcomes 2013;11:141.

[9] Legro RS, Chen G, Kunselman AR, Schlaff WD, Diamond MP, Coutifaris C, et al. Smoking in infertile women with polycystic ovary syndrome: baseline validation of self-report and effects on phenotype. Hum Reprod 2014;29(12):2680-6.

[10] Amer SA, Smith J, Mahran A, Fox P, Fakis A. Double-blind randomized controlled trial of letrozole versus clomiphene citrate in subfertile women with polycystic ovarian syndrome. Hum Reprod 2017;32(8):1631-8.

[11] Thessaloniki EA-SPCWG. Consensus on infertility treatment related to polycystic ovary syndrome. Hum Reprod 2008;23(3):462-77.

[12] Brown J, Farquhar C, Beck J, Boothroyd C, Hughes E. Clomiphene and anti-oestrogens for ovulation induction in PCOS. The Cochrane database of systematic reviews 2009(4):Cd002249.

[13] Legro RS, Brzyski RG, Diamond MP, Coutifaris C, Schlaff WD, Casson P, et al. Letrozole versus clomiphene for infertility in the polycystic ovary syndrome. The New England journal of medicine 2014;371(2):119-29.

[14] Zhang J, Si Q, Li J. Therapeutic effects of metformin and clomiphene in combination with lifestyle intervention on infertility in women with obese 
polycystic ovary syndrome. Pakistan journal of medical sciences 2017;33(1):8-12.

[15] Reefhuis J, Honein MA, Schieve LA, Rasmussen SA. Use of clomiphene citrate and birth defects, National Birth Defects Prevention Study, 1997-2005. Hum Reprod 2011;26(2):451-7.

[16] Run-Ming Y. The origin and development of chinese acupuncture and moxibustion. Ancient science of life 1985;4(4):224-8.

[17] Moini Jazani A, Nasimi Doost Azgomi H, Nasimi Doost Azgomi A, Nasimi Doost Azgomi R. A comprehensive review of clinical studies with herbal medicine on polycystic ovary syndrome (PCOS). Daru 2019;27(2):863-77.

[18] Wang Q, Deng H, Cheng K, Huang Z, Yin X, Zhou Y, et al. Manual acupuncture for the infertile female with polycystic ovary syndrome (PCOS): study protocol for a randomized sham-controlled trial. Trials 2019;20(1):564.

[19] Z J, Z B-x, L L. Acupuncture and moxibustion. Beijing: People's Medical Publishing House; 2014.

[20] Hui KK, Nixon EE, Vangel MG, Liu J, Marina O, Napadow V, et al. Characterization of the "deqi" response in acupuncture. BMC complementary and alternative medicine 2007;7:33

[21] Yang J, Liu Y, Huang J, Xu J, You X, Lin Q, et al. Acupuncture and Chinese medicine of artificial cycle therapy for insulin resistance of polycystic ovary syndrome with phlegm damp type and its mechanism. Zhongguo zhen jiu = Chinese acupuncture \& moxibustion 2017;37(11):1163-8.

[22] Min. Z, Hong-Yan. Y, Xiao-Yun. W, Jing-Jing. D, Jian. L. Randomized Controlled Study of Disease-syndrome Combination Therapy for Anovulatory Infertility. J Guangzhou Univ Tradit Chin Med 2017;34(06):815-8.

[23] Johansson J, Redman L, Veldhuis PP, Sazonova A, Labrie F, Holm G, et al. Acupuncture for ovulation induction in polycystic ovary syndrome: a randomized controlled trial. American journal of physiology Endocrinology and metabolism 2013;304(9):E934-43.

[24] Xu J, Zuo Y. Efficacy of acupuncture as adjunctive treatment on infertility patients with polycystic ovary syndrome. Zhongguo zhen jiu $=$ Chinese acupuncture \& moxibustion 2018;38(4):358-61.

[25] Yin Y, Zhang Y, Zhang H, Jiang D, Guo G. Clinical therapeutic effects of acupuncture combined with Chinese herbal medicine on infertility of polycystic ovary syndrome in the patients with ovulation induction with letrozole. Zhongguo zhen jiu = Chinese acupuncture \& moxibustion 2018;38(1):27-32.

[26] Yuan BC, Ma K, Zhang CH, Yuan Y. Bushen Huoxue herbal medicine in subfertile women with polycystic ovary syndrome: a Meta-analysis. Zhongguo Zhong Yao Za Zhi 2019;44(6):1080-6.

[27] Liao WT, Chiang JH, Li CJ, Lee MT, Su CC, Yen HR. Investigation on the Use of Traditional Chinese Medicine for Polycystic Ovary Syndrome in a Nationwide Prescription Database in Taiwan. Journal of clinical medicine 2018;7(7).

[28] Chao-chao Y, Li-hong K, Chao-yang M, Feng S, Guo-Jin Y, Yuan X, et al. The 
rules of acupoint-selection of acupuncture for polycystic ovary syndrome based on data mining World Journal of Acupuncture-Moxibustion (WJAM) 2016;26(03):73-8.

[29] Ge X, Yue-lai C. Regularities in Application of Acupoints in Acupuncture Treatment for Ovulatory Disorder Infertility Shanghai J Acu-mox 2017;36(5):620-5.

[30] Jin X, Chun-chun J, Zhen-ru S, Jie S, You-bing X. Study on Acupoint Seleection Rules of Treating Infertility in Ancient Literature. Journal of Nanjing University of Traditional Chinese Medicine 2019;35(6):719-23.

[31] Jo J, Lee YJ, Lee H. Acupuncture for polycystic ovarian syndrome: A systematic review and meta-analysis. Medicine 2017;96(23):e7066.

[32] Yun L, Liqun W, Shuqi Y, Chunxiao W, Liming L, Wei Y. Acupuncture for infertile women without undergoing assisted reproductive techniques (ART): A systematic review and meta-analysis. Medicine 2019;98(29):e16463.

[33] Lim CED, Ng RWC, Cheng NCL, Zhang GS, Chen H. Acupuncture for polycystic ovarian syndrome. The Cochrane database of systematic reviews 2019;7:CD007689.

[34] Wu XK, Stener-Victorin E, Kuang HY, Ma HL, Gao JS, Xie LZ, et al. Effect of Acupuncture and Clomiphene in Chinese Women With Polycystic Ovary Syndrome: A Randomized Clinical Trial. JAMA 2017;317(24):2502-14.

[35] Wang Y, Zhao Y, Yu S, Hu Y. Some issues from Effect of acupuncture and clomiphene in Chinese women with polycystic ovary syndrome in JAMA. Zhongguo zhen jiu = Chinese acupuncture \& moxibustion 2017;37(12):1342-6.

[36] Gang WJ, Jing XH. Is acupuncture actually not effective for polycystic ovary syndrome? : Analysis of the trial published in jama. Chin $\mathrm{J}$ Integr Med 2017;23(12):883-6.

[37] Gang WJ, Meng X, Jing XH. Assessing the adequacy of acupuncture in clinical trials: current status and suggestions. Zhongguo zhen jiu $=$ Chinese acupuncture $\&$ moxibustion 2019;39(3):229-33.

[38] Shi GX, Yang XM, Liu CZ, Wang LP. Factors contributing to therapeutic effects evaluated in acupuncture clinical trials. Trials 2012;13:42.

[39] Cao Y, Zhang L, Zhao D, Liu Z. DONG's extraordinary acupoints for the ovarian function of polycystic ovary syndrome:a randomized controlled pilot trial. Zhongguo zhen jiu $=$ Chinese acupuncture \& moxibustion 2017;37(7):710-4.

[40] Wang SJ, Zhang JJ, Qie LL. Acupuncture Relieves the Excessive Excitation of Hypothalamic-Pituitary-Adrenal Cortex Axis Function and Correlates with the Regulatory Mechanism of GR, CRH, and ACTHR. Evidence-based complementary and alternative medicine : eCAM 2014;2014:495379.

[41] Adeniji AA, Essah PA, Nestler JE, Cheang KI. Metabolic Effects of a Commonly Used Combined Hormonal Oral Contraceptive in Women With and Without Polycystic Ovary Syndrome. Journal of women's health (2002) 2016;25(6):638-45. 
Table 1. Baseline and clinical characteristics of the study population

\begin{tabular}{|c|c|c|c|}
\hline Characteristic & MA group $(n=41)$ & SA group $(n=38)$ & $p$ \\
\hline Age, year ${ }^{\dagger}$ & $30.8 \pm 3.5$ & $29.7 \pm 3.2$ & 0.918 \\
\hline Duration of illness, month ${ }^{\dagger}$ & $23.5 \pm 12.2$ & $22.0 \pm 8.5$ & 0.34 \\
\hline $\mathrm{BMI}^{\dagger}$ & $18.8 \pm 2.3$ & $17.9 \pm 2.1$ & 0.611 \\
\hline PCOS Scores $^{\dagger}$ & $3.6 \pm 1.6$ & $3.7 \pm 1.9$ & 0.371 \\
\hline TCM Scores $^{\dagger}$ & $4.9 \pm 2.4$ & $4.7 \pm 2.3$ & 0.954 \\
\hline \multicolumn{4}{|l|}{ Serum sex hormones level } \\
\hline $\mathrm{E}_{2}, \mathrm{pmol} / \mathrm{L}$ & $125.0(103.5,169.5)$ & $163.3(104.5,201.5)$ & 0.285 \\
\hline $\mathrm{T}, \mathrm{nmol} / \mathrm{L}$ & $1.6(1.2,2.0)$ & $1.4(1.0,2.1)$ & 0.283 \\
\hline $\mathrm{P}, \mathrm{nmol} / \mathrm{L}$ & $0.8(0.5,1.0)$ & $0.9(0.6,1.4)$ & 0.325 \\
\hline FSH, IU/L & $5.4(4.3,6.0)$ & $5.0(4.3,6.1)$ & 0.765 \\
\hline LH, IU/L & $6.1(3.1,9.6)$ & $4.2(2.5,7.0)$ & 0.116 \\
\hline
\end{tabular}

Abbreviations: MA group, manual acupuncture group; SA group, sham acupuncture group; BMI, body mass index, is the weight in kilograms divided by the square of the height in meters; PCOS, polycystic ovary syndrome; TCM, traditional Chinese medicine; $\mathrm{E}_{2}$, estradiol; T, total testosterone; $\mathrm{P}$, progesterone; FSH, follicle-stimulating hormone; LH, luteinising hormone;

${ }^{\dagger}$, Mean (SD);

$\ddagger$, Median (IQR). 
Table 2. Comparison of pregnancy rates between the two groups During the Entire Study $\quad$ N $(\%)$

\begin{tabular}{lllll}
\hline Groups & $\mathrm{N}$ & After treatment & During follow-up & Total \\
\hline MA group & 41 & $10(24.39)$ & $9(29.03)$ & $19(46.34)$ \\
SA group & 38 & $5(13.16)$ & $2(6.06)$ & $7(18.42)$ \\
$p$ & - & 0.203 & 0.015 & 0.008 \\
\hline
\end{tabular}

Abbreviations: MA group, manual acupuncture group; SA group, sham acupuncture group;

Table 3. Comparison of ovulation rate between the two groups (number of times)

\begin{tabular}{lllll}
\hline Groups & Ovulation & No ovulation & Ovulation rate & $p$ \\
\hline MA group & 75 & 54 & $58.14 \%$ & \\
SA group & 59 & 70 & $45.74 \%$ & 0.046 \\
\hline
\end{tabular}

Abbreviations: MA group, manual acupuncture group; SA group, sham acupuncture group; 
Table 4. Comparison of other secondary outcomes before and after treatment in the two groups $(\bar{x} \pm s) /[M(Q 25, Q 75)]$

\begin{tabular}{|c|c|c|c|c|c|c|c|}
\hline Items & Groups & $\mathrm{N}$ & Baseline & After treatment & $\begin{array}{l}p \text { value } \\
\text { (intra-group) }\end{array}$ & $\begin{array}{l}\text { Index value reduction } \\
\text { change }{ }^{\mathrm{b}}(\%)\end{array}$ & $\begin{array}{c}p \text { value } \\
\text { (between } \\
\text { groups) }\end{array}$ \\
\hline \multirow[t]{2}{*}{ PCOS Score } & MA group & 31 & $3.71 \pm 1.75$ & $2(0,3)$ & $<0.001$ & $60(33.33,100)$ & 0.003 \\
\hline & SA group & 33 & $3.64 \pm 1.85$ & $2(1.5,3)$ & $<0.001$ & $33.33(0,100)$ & \\
\hline \multirow[t]{2}{*}{ TCM Score } & MA group & 31 & $5.0 \pm 2.61$ & $2(1,3)$ & $<0.001$ & $60(50,71.43)$ & 0.317 \\
\hline & SA group & 33 & $4.67 \pm 2.33$ & $2(1,3)$ & $<0.001$ & $60(46.43,66.67)$ & \\
\hline \multirow[t]{2}{*}{$\mathrm{E}_{2}(\mathrm{pmol} / \mathrm{L})$} & MA group & 31 & $131(107,167)$ & $119.33 \pm 52.31$ & 0.009 & $18.29(-2.9,42.8)$ & 0.131 \\
\hline & SA group & 33 & $161.48(90.5,203)$ & $132(77.5,187.5)$ & 0.513 & $-6.9(-18.44,34.48)$ & \\
\hline \multirow[t]{2}{*}{$\mathrm{T}(\mathrm{nmol} / \mathrm{L})$} & MA group & 31 & $1.59(1.32,2.03)$ & $1.15(0.95,1.57)$ & $<0.001$ & $23.77 \pm 22.99$ & 0.037 \\
\hline & SA group & 33 & $1.15(0.95,2.08)$ & $1.14(0.87,1.68)$ & 0.131 & $2.13(-14.58,35.29)$ & \\
\hline \multirow[t]{2}{*}{$\mathrm{P}(\mathrm{nmol} / \mathrm{L})$} & MA group & 31 & $0.8(0.5,1.0)$ & $0.6(0.4,0.79)$ & 0.023 & $20(0,40)$ & 0.824 \\
\hline & SA group & 33 & $0.8(0.59,1.33)$ & $0.6(0.4,0.9)$ & 0.008 & $14.26 \pm 50.344$ & \\
\hline \multirow[t]{2}{*}{ FSH(IU/L) } & MA group & 31 & $5.26 \pm 1.08$ & $5.08 \pm 1.26$ & 0.368 & $1.80 \pm 21.42$ & 0.92 \\
\hline & SA group & 33 & $5.04(4.27,6.23)$ & $5.02(4.19,5.81)$ & 0.561 & $1.98(-16.66,16.61)$ & \\
\hline \multirow[t]{2}{*}{ LH(IU/L) } & MA group & 31 & $6.89 \pm 4.01$ & $3.94(2.48,6.58)$ & 0.043 & $13.99(-13.7,55.33)$ & 0.108 \\
\hline & SA group & 33 & $4.17(2.50,6.83)$ & $4(2.59,6.84)$ & 0.888 & $0(-69,35.4)$ & \\
\hline \multirow[t]{2}{*}{ LH/FSH } & MA group & 31 & $1.35 \pm 0.75$ & $0.82(0.55,1.3)$ & 0.038 & $20.88(-17.02,39.78)$ & 0.149 \\
\hline & SA group & 33 & $0.84(0.56,1.34)$ & $0.83(0.62,1.30)$ & 0.728 & $-0.17(-47.18,33.42)$ & \\
\hline \multirow[t]{2}{*}{ HOMA-IR } & MA group & 31 & $2.06(1.32,2.56)$ & $1.7(1.18,2.7)$ & 0.753 & $-2.62 \pm 40.53$ & 0.736 \\
\hline & SA group & 33 & $1.69(1.15,2.71)$ & $1.86(1.22,3)$ & 0.845 & $0(0,8.15)$ & \\
\hline
\end{tabular}


${ }^{\mathrm{b}}$ Index value reduction change $=($ After treatment-Baseline $) /$ Baseline $\mathrm{x} 100 \%$.

Abbreviations: MA group, manual acupuncture group; SA group, sham acupuncture group; PCOS, Polycystic Ovary Syndrome; TCM,

Traditional Chinese Medicine; E2, estradiol; T, total testosterone; P, progesterone; FSH, follicle-stimulating hormone; LH, luteinising hormone;

HOMA-IR=fasting blood glucose $\times$ fasting insulin/22.5. 
